\title{
Superconductivity of Very Thin Films: The Superconductor-Insulator Transition
}

\author{
Yen-Hsiang $\operatorname{Lin}^{1}$, J. Nelson, A. M. Goldman \\ School of Physics and Astronomy, University of Minnesota, 116 Church Street SE, Minneapolis, Minnesota 55455, USA
}

\begin{abstract}
The study of thin superconducting films has been an important component of the science of superconductivity for more than six decades. It played a major role in the development of currently accepted views of the macroscopic and microscopic nature of the superconducting state. In recent years the focus of research in the field has shifted to the study of ultrathin films and surface and interface layers. This has permitted the exploration of one of the important topics of condensed matter physics, the superconductor-insulator transition. This review will discuss this phenomenon as realized in the study of metallic films, cuprates, and metallic interfaces. These are in effect model systems for behaviors that may be found in more complex systems of contemporary interest.
\end{abstract}

Keywords: Superconductor-Insulator, Quantum Phase Transition, SIT

\section{Introduction}

The subject of thin film superconductivity is quite broad, and if treated in detail would encompass several monograph volumes. In this review we will limit the discussion to the superconductor-insulator transition. Superconductor-insulator transitions (SITs) in two dimensions, especially those implemented in thin films and interfaces, can provide the simplest examples of the continuous quantum phase transition paradigm[1]. Quantum phase transitions differ from thermal phase transitions in that they occur at zero temperature and involve a change of the ground state in response to the variation of an external parameter of the Hamiltonian. In the example of the superconductor-insulator transition, this control parameter could be a parallel or perpendicular magnetic field, disorder, or charge density. Quantum phase transitions are studied through measurements at nonzero temperature of physical behaviors influenced by the quantum fluctuations associated with the zero-temperature transition that persist at nonzero temperatures. Here we focus on aspects of superconductor-insulator transitions in films that are effectively two-dimensional insofar as their superconducting properties are concerned. In addition to work on disordered thin films, recent developments such as the superconductor-insulator transitions observed in the metallic layer found at interfaces between certain different insulators, and those associated with the electrostatic tuning of high temperature superconductors will be included. We will not discuss transitions in either three-dimensional systems [2] or Josephson junction arrays [3].

\footnotetext{
Email address: goldman@physics.umn.edu (A. M. Goldman)

${ }^{1}$ Current Address: Department of Physics, University of Maryland College Park, MD 20742-4111
}

Quantum phase transitions are studied through measurements at nonzero temperature of physical behaviors influenced by quantum fluctuations. Among other systems exhibiting quantum phase transitions are ${ }^{4} \mathrm{He}$ adsorbed on random substrates, two-dimensional electron gases, and numerous complex, strongly correlated electron materials. The concept is also relevant to the physics of cold atoms, which in some instances can serve as model systems for many condensed matter phenomena[4]. Superconductorinsulator transitions are of particular importance because of their connection with the fundamental phase-number uncertainty relation, which makes them the simplest of such transitions. Although the subject of SITs might be considered to be mature, there remain many open issues resulting from the study of new materials, the application of new experimental techniques, and the continued extension of measurements to lower temperatures and higher magnetic fields. Although there are common observations in diverse experiments involving rather different types of materials, there are dramatically contrasting results in others involving similar materials and similar measurements. In some studies there is a direct transition between the insulating and superconducting regimes as a function of the control parameter, whereas in others, there is evidence of the spontaneous appearance of inhomogeneity near the transition, which may be manifested as an intermediate metallic regime or a breakdown of scaling as zero temperature is approached. The nature of the insulating state (or localizing quantum-corrected metallic state) is a subject of ongoing research. Not all of the magnetic field tuned SITs exhibit a giant magnetoresistance peak above the critical field for the SIT and although there has been progress, there is of yet no consensus as to its explanation in those circumstances in which it is found. The grouping 
of the various transitions into two distinct groups, bosonic and fermionic, also appears to be in need of refinement.

\section{Disordered Materials and Tuning Parameters for the SIT}

Experiments on various materials with different tuning parameters do not clearly distinguish between competing theoretical pictures. Indeed there may be several types of SI transitions. First, the mechanism may depend upon the tuning parameter, which can be disorder, electrostatic charge, perpendicular or parallel magnetic field, surface magnetic impurities, or dissipation. Second, the mechanism and phase diagram may depend upon the type of system being investigated, $i$. e., whether it is an ultrathin quench condensed amorphous or granular film, an amorphous film of $\mathrm{InO}_{x}, \mathrm{MoGe}, \mathrm{Nb}_{0.15} \mathrm{Si}_{0.85}$, or Ta, a polycrystalline film of TiN, a monolayer or ultrathin film of a high temperature superconductor, or a conducting interface separating two band insulators. Third, in the case of films, the character of the substrate may matter. The nature of the transition could be altered in the presence of a high dielectric constant substrate such as $\mathrm{SrTiO}_{3}$, or may depend upon the nature of an underlayer. The roughness of the substrate may serve as a template for the roughness of the film, which in turn may control the character of the transition. Finally another issue is the role of spin-orbit scattering. Experiments on high-Z elements ( $a$-Bi or $a$ $\mathrm{Pb}$ ) may produce different results from those carried out on low- $\mathrm{Z}$ elements $(a-\mathrm{Be}$ or $\mathrm{Al})$, where there is minimal spin-orbit scattering.

Among some of the phenomena that have sparked continuing interest in the field are the giant magnetoresistance peak found in some nearly critical SIT systems, leading in some instances to what has been called "superinsulating" behavior at sufficiently low temperatures $[5,6]$. In some systems there appears to be a "quantum metal" in the limit of very high magnetic fields[7, 8]. A deep analogy may exist between the insulating regime of some of these systems and the pseudogap regime of the high temperature superconductors $[9,10]$. Other issues include the possibility of an intermediate metallic phase between the superconductor and insulator in some perpendicular magnetic field tuned transitions[11].

The nature of the insulating regime, apart from the magnetoresistance peak, is a critical issue. There is experimental evidence from a variety of sources that for some, but not all systems, superconducting coherence may persist over finite length scales in the insulating regime[12, 13, 14, 15]. The Nernst effect has emerged as a means of exploring the insulating regime of magnetic field-tuned SITs[16, 17, 18].

The possibility of electrostatically inducing superconductivity has been demonstrated, allowing the exploration of behavior that does not depend on possible changes in morphology associated with different doping or film thickness $[19,20]$. Using electrostatic charging or doping it is possible to import into the study of the SIT some of the approaches used in 2D electron gas physics. Very recent additions to this direction of research have been the use of electric double layer transistors (ELDTs) employing ionic liquids to electrostatically gate the SIT of monolayers[21] and ultrathin films [22,23] of high temperature superconductors and the gating of superconductivity at the interface between insulators[24, 25].

\section{Theoretical Scenarios for the SIT of Disordered Materials}

Interest in SI transitions arose in the context of study of the interplay between superconductivity and disorder. Anderson[26] and Abrikosov and Gor'kov[27] showed that nonmagnetic impurities don't affect the superconducting transition temperature because Cooper pairs form from extended time-reversed eigenstates, which have disorder included. On the other hand with sufficient disorder Anderson localization occurs, and superconductivity will not develop, even in the presence of an attractive electronelectron attraction [28]. This leads to the question of how superconductivity disappears with increasing disorder $[29,30,31,32,33,34]$. At nonzero temperatures, the superconducting phase transition is a continuous phase transition, so that there are fluctuations. For a transition suppressed to zero temperature, the fluctuations become quantum in character and the transition becomes a quantum phase transition.

In two-dimensions (2D) there is added complication as the superconducting transition at nonzero temperatures is a topological, or Berezinskii-Kosterlitz-Thouless (BKT) transition[35]. Furthermore all 2D electronic systems were initially believed to be localized, even for arbitrarily weak disorder. This changed with the observation of apparent metal-insulator transitions in strongly-interacting, high-mobility low-carrier-density MOSFETs[36], although the "metallic" state in MOSFETs is still under debate. Superconductor-insulator transitions in 2D are additional examples in which interactions play a critical role.

There have been four general approaches to explaining the demise of superconductivity in the $2 \mathrm{D}$ limit as a function of disorder or magnetic field. The first is based on a perturbative microscopic description of homogeneous systems and considers the interplay between the attractive and repulsive electron-electron interactions in the presence of disorder. In this microscopic description, suppression of the critical temperature follows from the renormalization of the electron-electron interaction in the Cooper channel by the long-range Coulomb repulsion in the presence of disorder[37, 38, 39]. The amplitude of the superconducting order parameter is suppressed by disorder, and at a sufficient level of disorder, Cooper pairs break up into fermions, which localize in $2 \mathrm{D}$. This is the so-called fermionic picture. The second approach is a boson localization theory. Inside the critical region, Cooper pair phase fluctuations are important, and these pairs in some approximation are bosons [40]. The observation of apparently direct SI transitions in 


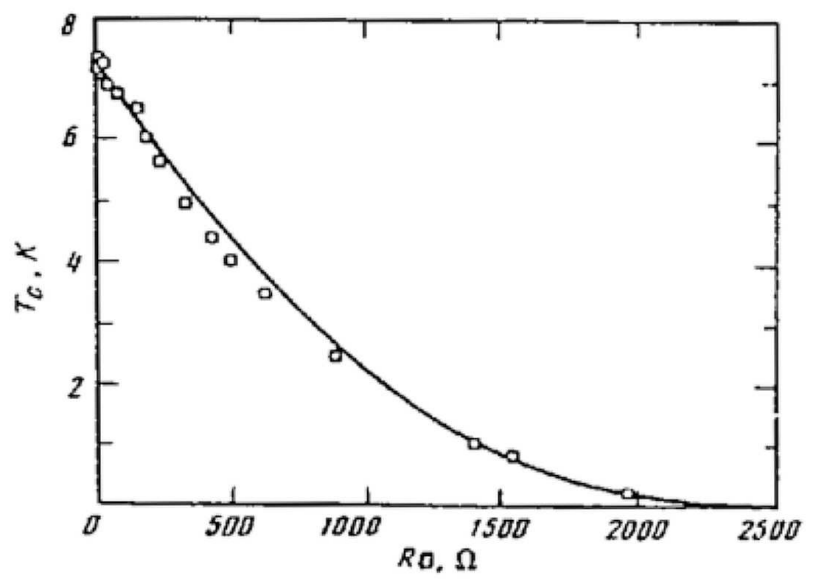

Figure 1: Suppression of superconductivity in amorphous $\mathrm{Mo}_{x} \mathrm{Ge}_{y}$ with increasing disorder (Ref. [45]). the solid line is a theoretical fit as discussed in Ref. [39].

ultrathin films, in the $\mathrm{T} \rightarrow 0$ limit $[41,42]$, in part served to motivate this approach. This will be discussed below. The third is based on the physics of granular superconductors. It builds on dissipative models of resistively shunted Josephson junction arrays, and may have application to both granular and homogeneous 2D superconductors[43]. The fourth involves percolation[44], which may play a role in all of the other pictures if the samples are even slightly inhomogeneous, which is generally unavoidable in the real world. The message conveyed by experiment regarding the applicability of various models continues to be quite mixed and indeed the explanation of some transitions may involve more than one idea.

As long as the reduction of the transition temperature is relatively small, the perturbative approach mentioned above should provide an appropriate representation of the physics. In the regime where the transition would be suppressed to zero there will be quantum fluctuations, and this theory should fail, as such fluctuations are not included[43]. On the other hand, the reduction of the mean field transition temperature that is predicted by perturbative theory based on the weakening of the screening of the Coulomb interaction with increasing disorder[39] is consistent with studies of homogeneous a-MoGe films by Graybeal and Beasley [45]. (See Fig. 1.)

This scenario would apply to disordered systems, but might be expected to ultimately break down. Alternatively the scenario could be modified at sufficiently low temperatures because of the onset of the previously mentioned quantum fluctuations. There may be alternate fermionic scenarios in electrostatically doped SITs where there is evidence in both disordered systems and in high temperature superconductors of a dramatic change in the electronic properties at the SIT. In the case of high temperature superconductors the transition may even involve changes in the electronic structure. These issues will be discussed later.
Boson localization theories [40, 46, 47] are closely related to models used to describe superfluid helium and Josephson junction arrays. Doniach[48] pioneered this approach, which involves phase fluctuations, in the context of Josephson junction arrays. There is a quantum critical point, and the ground state can be controlled by disorder or perpendicular magnetic field. An important prediction is that at the critical level of disorder, or magnetic field, Cooper pairs move diffusively, leading to metallic behavior in the $T \rightarrow 0$ limit. This occurs only at the quantum critical point. If one assumes complete duality between Cooper pairs and vortices[49], this resistance is universal with a value given by the quantum resistance for pairs, $h / 4 e^{2}$. The physics is related to the universal jump condition in the finite temperature BKT transition[35].

The first real indication that disorder might drive the transition temperature down to a low enough value that quantum fluctuations could be important was the work of Haviland, Liu and Goldman [41] in which the thickness variation of resistance vs. temperature $R(T)$, was studied in $a$-Bi films. Bismuth is a semimetal in its usual form, but is metallic and superconducting under high pressure, or in thin film amorphous form, which results when it is grown on substrates held at liquid helium temperatures[30]. The experiments were performed in an apparatus in which the films could be grown in situ, with the substrates held at liquid helium temperatures and in which measurements carried out in alternation with increments of growth. If such studies are carried out using substrates pre-coated with a thin layer of amorphous $\mathrm{Ge}$ or Sb, atomic scale rather than mesoscopic disorder results.

In contrast, films made using a low temperature evaporation technique but without an underlayer of amorphous $\mathrm{Ge}$ or $\mathrm{Sb}$, are granular in that they possess mesoscopic clusters. They also exhibit a separation between superconducting and insulating behavior in the limit of zero temperature, but the properties of these films differ in detail from those of the nominally homogeneous amorphous films described above. In particular the dependence of the resistance vs. temperature can be a nonmonotonic function of temperature, with the appearance of local minima in $R(T)$ which deepen as films are made thicker, ultimately giving rise to a clean resistive superconducting transition [50]. A detailed theory of this behavior does not exist, partially as a consequence of large sample-to-sample variations in behavior due to uncontrolled inhomogeneity. In the superconducting state of polycrystalline $\mathrm{Sn}$ thin films, $T_{c}$ was observed to oscillate with thickness[51]. In addition these oscillations have recently been observed in crystalline $\mathrm{Pb}$ films grown epitaxially[52].

Nominally homogeneous films have been the subject of considerable study. Curves of $R(T)$ for a sequentially deposited set of $a$-Bi films grown on $a$-Ge substrates are presented in Fig. 2. These resemble renormalization flows to an unstable fixed point at zero temperature, which for this particular set of films corresponds to the quantum resistance for pairs, $h / 4 e^{2}$, or $6450 \Omega / \square$. This result set 


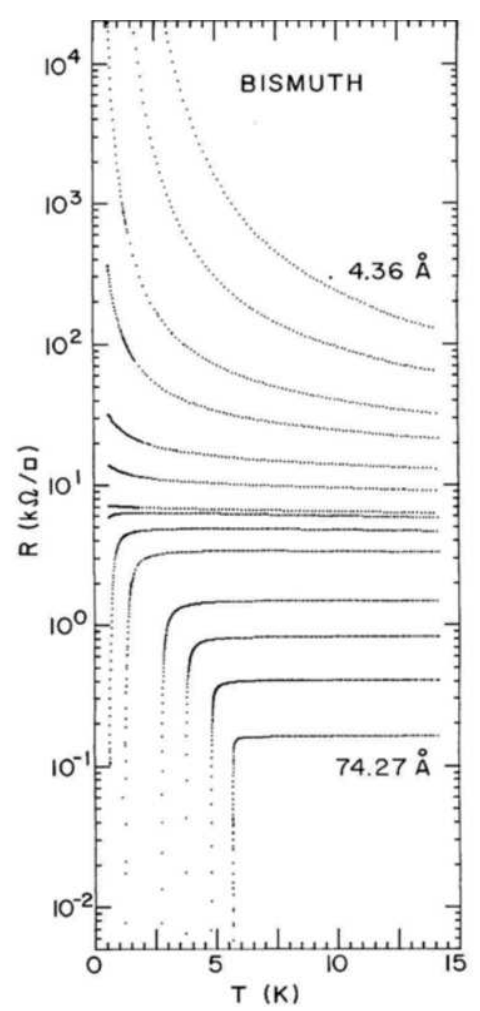

Figure 2: Evolution of the temperature dependence of the logarithm of the sheet resistance $R(T)$ with thickness for an $a$-Bi film deposited onto a $5 \AA$ thick layer of $a$-Ge. The measurements were alternated with incremental evaporations of Bi. All of the increments of film thickness including the underlayer were deposited without breaking vacuum and without warming above liquid helium temperatures. Fewer than half of the traces actually acquired are shown. Film thicknesses that are shown range from 4.36 to $74.27 \AA$. (From Ref. [41])

the stage for consideration of the SIT as a continuous quantum phase transition and motivated the application of Boson physics to the SIT. Unfortunately subsequent studies of the SIT in other materials indicated that the critical resistance was not universal at $h / 4 e^{2}$. Yazdani and Kapitulnik[53] in their study of the magnetic fielddriven SIT, suggested that the nonuniversal critical point resistance could be a consequence of a parallel fermionic conductance channel at nonzero temperature, a view also supported by Gantmakher and collaborators[54]. However this approach does not describe all of the systematics of the data of Markovic et al, $[55,56]$ on the magnetic fielddriven transition of quench-evaporated $a$-Bi films of different thicknesses.

Without a duality assumption, and with a realistic form for the interaction between charges, the resistance at the critical point is a nonuniversal constant. Experimentally the resistance appears to be sample-dependent but is within a factor of 2 or $1 / 2$ of the quantum resistance for pairs[57], although there is work[58, 59] that suggests that there may be regimes in which the critical resistance of the magnetic field-driven SI transition may indeed be $h / 4 e^{2}$. In elaborations of the Bose-Hubbard model, the value of the critical resistance depends on the form of interaction between charges, and the details of the calculations. (See for example Refs. [60, 61, 62, 64, 65].) Some of these works are concerned with including low energy quasiparticle interactions in the theory. These are claimed to be a most serious aspect of missing physics, and they introduce amplitude fluctuations into the description of the transition.

Tunneling experiments on quench evaporated homogeneous films of metals similar in preparation to those shown in Fig. 2 seem to support fermionic rather than bosonic scenarios [66]. The mean field transition temperature and the energy gap are suppressed at a fixed ratio as sheet resistance increases, a phenomenon treated theoretically by Smith et al.[67]. The energy gap also vanishes on the insulating side of the SIT of homogeneously disordered films [68], and on the insulating side of the field-tuned transition[69]. These studies suggest that amplitude fluctuations and not phase fluctuations drive the SIT. The reason for this interpretation is that the amplitude of the order parameter is vanishing with the energy gap in the insulating regime, which would appear to falsify the Bosonlocalization model. On the other hand, contemporary tunneling measurements on $\mathrm{InO}_{x}$ films indicate the presence of a gap, but no coherence peak on the insulating side of the disorder-tuned SIT[70, 71]. This would imply the existence of Cooper pairs in the insulating regime.

It is essential to reconcile these disparate results. First it should be noted that quench evaporated films are very different in morphology and chemistry from disordered $\mathrm{InO}_{x}$ films. Second, drawing a conclusion that the vanishing of the gap in the insulating regime implies the absence of Cooper pairs should be treated with care as the vanishing of the gap feature in superconducting tunneling could result from other effects such as pair breaking by disorder[72]. More will be said later regarding the differences between disordered films of $\mathrm{InO}_{x}$ and TiN, and quench-evaporated films.

\section{Scaling Analyses of Continuous SITs}

Because the SIT in many instances appears to be a direct continuous quantum phase transition, it is of interest to analyze data within that framework. As mentioned, the application of the quantum phase transition (QPT) paradigm to the SIT followed the publication of the data of Fig. 2. Continuous QPTs are transitions at absolute zero brought about by changing a parameter in the Hamiltonian of the system $[1,73]$. The quantum mechanical ground state changes when the critical point is crossed. In contrast with phase transitions at nonzero temperature, quantum effects are central to QPTs. In other phase transitions, although the order parameter itself may be quantum mechanical, classical thermal order parameter fluctuations govern its behavior at the relevant long wavelengths. In the case of QPTs, the fluctuations themselves are quantum mechanical. Near a QPT there are divergent correlation lengths, for the spatial, $\xi$, and temporal, 
$\xi_{\tau}$, directions. The latter is associated with a vanishing energy scale. These lengths depend on the control parameter, $\delta=\left(g-g_{c}\right) / g_{c}$ for the transition. Here $g$ can be a measure of disorder, magnetic field, or charge, and $g_{c}$ is the critical value of the control parameter. We then write $\xi \propto|\delta|^{\nu}$ and $\xi_{\tau} \propto \xi^{z}$. This defines the correlation length exponent $\nu$ and the dynamical critical exponent $z$. The exponents of the correlation length of the quantum phase transition, $\xi$, and the dynamical correlation length, $\xi_{\tau}$, are believed to be independent of the microscopic details of the transition and depend only on properties of the system such as the symmetry breaking associated with the transition, the dimensionality of the system, and the range of the interactions. These determine the universality class of the quantum phase transition. Physical quantities in the critical region close to the $\mathrm{QPT}$ are homogeneous functions of the independent variables in the problem.

A key feature of QPTs is the interplay of dynamics and thermodynamics. As a consequence of this, a $d$ dimensional quantum system at finite temperature is described in the $T \rightarrow 0$ limit as a classical system with the finite extent of the system in an extra dimension being given by $-\hbar \beta$ in units of time, where $\beta=1 / k_{B} T$. The extent of the extra dimension is divergent only in $T \rightarrow 0$ limit. What is remarkable is that the universality class of the quantum transition may be one studied extensively in some classical context. This also allows for the possibility of a computational treatment of the quantum mechanical problem using simulations of the $d+z$ dimensional classical problem. $[1,73]$ Disorder on the other hand can change the universality class of the equivalent classical problem. It is also not true in general that space and time enter in the same fashion in the equivalent classical problem. For this to happen the dynamical exponent, $z$, must be unity, and whether or not this happens depends upon the detailed quantum dynamics of the system.

As mentioned above, the effect of considering $T \neq 0$ in the statistical mechanics is to force the temporal dimension of the problem to be finite. The formal model used to analyze data at nonzero temperatures is finite-size scaling[73]. The success of finite size scaling analyses of the various superconductor-insulator transitions is part of the evidence for there being QPTs. Scaling can be used to characterize properties measured at nonzero temperatures in the regime of critical fluctuations and thus to determine the critical exponents and universality class of the transition. The resistance near the transition is of the form:

$$
R=R_{c} F\left(\delta / T^{1 / \nu z}, \delta / E^{1 / \nu(z+1)}\right)
$$

Here $R$ is the sheet resistance and $R_{c}$ is an arbitrary constant. The function $F$ is system specific. The energy scale for the fluctuation, $\Omega \propto \xi_{\tau}^{-z}$ is cut off at a nonzero temperature by $k_{B} T$, defining a cutoff length, $L_{T}$, given by $k_{B} T \sim L_{T}^{-Z}$. This gives rise to the first term in the argument of the function $F$. The second term comes from a characteristic length associated with the electric field as compared with the correlation length. The applicability of these ideas assumes that there is a continuous and direct SIT. Hebard and Paalanen carried out the first scaling analysis of a perpendicular field driven transition of $\mathrm{InO}_{x}$ films[42]. They found that the exponent product, $\nu z \approx 1.2$ from scaling with only the first argument of Eq. 1. Later Yazdani and Kapitulnik analyzed the properties of MoGe films, carrying out both temperature and electric field scaling[53]. They reported both $\nu z$ and $\nu(z+1)$ and thus ascertained that $z=1$ in agreement with expectations for systems with long-range Coulomb interactions[74].

For homogeneous films of different types, including cuprates, there are numerous finite-size scaling analyses of experimental data for the perpendicular field-driven transition in addition to the work of Hebard and Paalanen and Yazdani and Kapitulnik. These include Palaanen, Hebard, and Ruel [75], Okuma and Kokubo [76], Inoue et al. [77], Seidler, Rosenbaum and Veal [78], Wang, et al. [79], Okuna, Terashima and Kokubo [79, 80], Gantmakher et al. [54], and on the thickness and field-tuned transitions together, Markovic et al. $[55,56]$. These are all consistent with a quantum critical point. An important caveat is that the success of scaling by itself and the identification of the universality class of the transition from the values of the critical exponents may not elucidate the microscopic physics of the transition or the nature of the insulating state.

As an example of the above analysis, we consider data of $R(T, d)$ for a set of films different from those shown in Fig. 3 . Here $d$ is the film thickness which is sufficiently small so that it limits the mean free path. The normal state (high temperature) resistance would be an alternative control or tuning parameter. As is typical of such experiments, at some critical thickness, $d_{c}$, R becomes temperature independent, while for even thicker films it decreases rapidly with decreasing temperature, indicating the onset of superconductivity. The critical thickness is found by plotting $R$ vs. $d$ at different temperatures and identifying the crossing point which is the thickness at which the resistance is temperature independent. Alternatively one can plot $d R / d T$ vs. $d$ at the lowest temperatures and find the thickness for which $d R / d T \rightarrow 0$. In the quantum critical regime the resistance of a two-dimensional system is expected to obey the scaling form of Eq. 1, with the in-plane electric field taken to be a constant.

To analyze the data, we obtain curves of $R(d)$ at various temperatures. Having determined the critical thickness in the manner described above, we then rewrite Eq. 1 as $R(t, \delta)=R_{c}(\delta t)$ where $t=T^{-1 / \nu z}$, and treat the parametert $(T)$ as an unknown variable which is determined at each temperature to obtain the best collapse of the data. Specifically, $t$ is determined by performing a numerical minimization between a curve at a particular temperature and the lowest temperature curve. The exponent product $\nu z$ is then found from the temperature dependence of $t$, which must be a power law in temperature for the procedure to make physical sense. This approach 


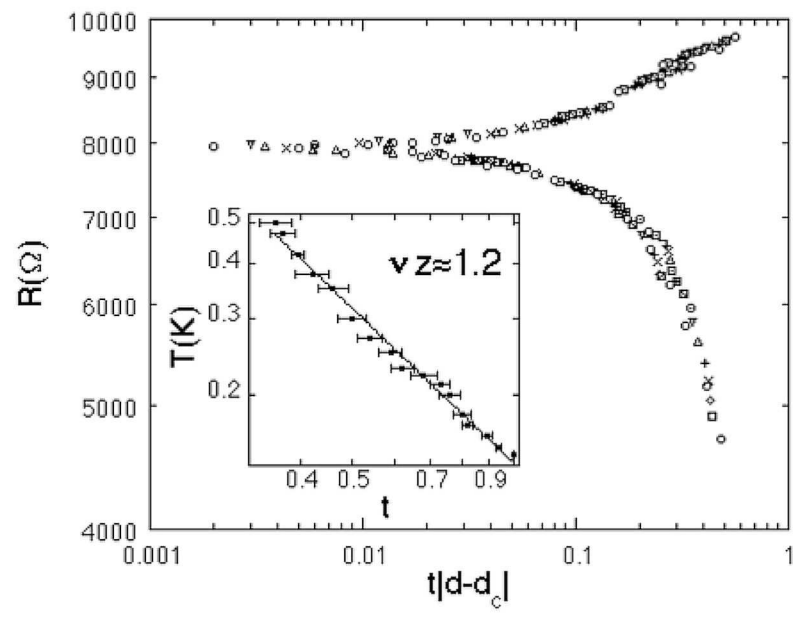

Figure 3: Resistance per square as a function of the scaling variable, $t\left|d-d_{c}\right|$, for seventeen different temperatures, ranging from 0.14 to $0.5 \mathrm{~K}$. Different symbols represent different temperatures. Inset: temperature dependence of $t$. (From Ref. [55])

does not require detailed knowledge of the functional form of the temperature- or thickness-dependence of $R$, or prior knowledge of the critical exponents. A different method of obtaining critical exponents was also used to check the consistency of this procedure. A log-log plot of $\partial R /\left.\partial d\right|_{d_{c}}$ vs. $T^{-1}$ was constructed. Its slope is equal to $1 / \nu z$ if Eq. 1 is obeyed. Exponents obtained this way are essentially identical to those obtained using the first procedure, within the quoted experimental uncertainty.

The collapse of the data of $R(T, d)$ is shown in Fig. 3 . The exponent-product $\nu z$ is found to be $1.2 \pm 0.2$, with the error determined from the power law fit. This is consistent with theoretical predictions in which $z=1$ would be expected for a bosonic system with long-range Coulomb interactions, and with $\nu>1$ in two dimensions. The latter corresponds to the expectation for a system, which is disordered, where the Harris criterion in the context of finite-size scaling specifies that $\nu \geq 2 / d[81]$.

When the exponent product was determined using a similar analysis on superconducting $a$-Bi films of fixed thicknesses, with perpendicular magnetic field rather than thickness as the control parameter, $\nu z=0.7 \pm 0.2$ was found. The fact that the field-tuned transition exponent product differs from the thickness-tuned, suggests that the transitions belong to two different universality classes. If $\mathrm{z}=1$, this result, with $\nu \sim 0.7$, would correspond to the $(2+1) \mathrm{D}$ XY model. The notion notes the addition of a temporal dimension to the $2 \mathrm{D}$ model. An exponent $\nu \sim 2 / 3$ corresponds to what is found in various calculations for the correlation length exponent of the $3 \mathrm{D} \mathrm{XY}$ model. The fact that $\nu \leq 2 / d$ in this instance suggests a clean limit.

It is important to note several features of these two SITs. The transitions are direct in that there appear to be no in- termediate metallic phases and there is no resistance saturation at the lowest temperatures. This is in contrast with results reported for other types of systems $[22,82]$ or what is found in the case of granular films [50]. It should also be noted that the films, which have been studied in the case of the perpendicular field-tuned SIT are very close in their properties to the insulating regime. Some studies of $a-\mathrm{Pb}$ films [83] have revealed the presence of an intermediate regime separating the insulating and superconducting regimes. The reason for this is not known at this time.

Parker et al. [83] also explored an SIT by decorating quench evaporated $a-\mathrm{Pb}$ films with magnetic impurities. They did not carry out a scaling analysis of this data, but were able to traverse the SIT. The SIT of $a$-Bi films has also been studied in parallel magnetic fields with two types of samples, those in which superconductivity was induced by electrostatic doping, which will be discussed below, and those which were intrinsic superconductors with thicknesses close to the critical thickness for the appearance of superconductivity [84]. In both instances exponent products of about 0.7 were found.

The electrostatic tuning of the SIT gets around the issues of changes of sample morphology with thickness and possible vortex pinning that could be important in both thickness and perpendicular magnetic field tuned transitions. In this approach the level of physical disorder is in principle fixed, and the outcome is not dependent upon the degree of vortex pinning. To effect electrostatic tuning of the SIT a field effect transistor geometry was employed in which a $\mathrm{SrTiO}_{3}$ (STO) crystal served as both the substrate and dielectric or gate insulator. A sequence of $a$-Bi films was prepared in the incremental manner described above [19, 84]. These films, once connected, were all insulating with the temperature dependence governed by the $2 \mathrm{D}$ Mott variable range hopping form $R=R_{0} \exp \left[\left(T_{0} / T\right)^{1 / 3}\right]$. The last film in this sequence had a nominal thickness of $10.22 \AA$. The addition of electrons to this film using the field effect induced superconductivity as shown in Fig. 4. An important finding in this work was a crossover from 2D Mott hopping in the insulating regime to a $\ln T$ dependence of the conductance on temperature in the normal state for films which underwent a transition to superconductivity at a nonzero temperature. What was also found is that the coefficient of the $\ln T$ term in the conductance was a linear function of the charge transfer, as shown in the inset to Fig. 4. This coefficient is related to the screening of the electronelectron interaction.

This SIT was successfully analyzed using finite-size scaling, employing the charge transfer $\Delta n$ as the control parameter. This suggests that the electrostatically tuned transition is a continuous quantum phase transition. In this instance, the exponent product $\nu z$ was $0.7 \pm 0.1$, including data all the way up to $1 \mathrm{~K}$ if the $\ln T$ dependence of the conductance was first removed. This was done by assuming that there were two parallel conductance channels, 


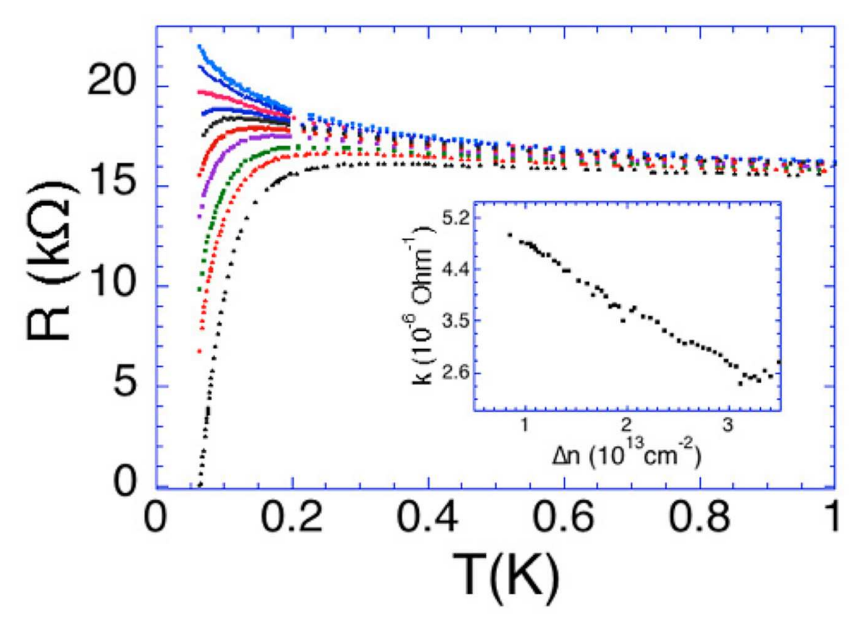

Figure 4: Resistance versus temperature at various values of $\Delta n$ for a $10.22 \AA$ thick $a$-Bi film with $B=0$. Data are shown from $60 \mathrm{mK}$ to $1 \mathrm{~K}$. The values of $\Delta n$ that are shown, from top to bottom, are $0,0.62,1.13,1.43,1.61,1.83,2.04,2.37,2.63$, and $3.35 \times 10^{13} \mathrm{~cm}^{-2}$. Forty four curves of $R(T)$ for other values of $\Delta n$ are omitted from the plot for clarity. Inset: slope of $\ln T$ vs. $\Delta n$ in the normal state. (From Ref. [19])

one involving weak localization and electron-electron interactions, and the other the critical fluctuations. The fact that an insulator-metal transition coincides with the insulator superconductor transition suggests that the chargetuned SI transition involves electronic phenomenon and is not associated with the localization of bosons.

\section{Metallic Regimes and Electron Heating Effects}

Measurements by Mason and Kapitulnik [85], Chervenak and Valles [86], Qin et al. [87], and Seo et al. [11] support the idea of a metallic regime for certain MoGe and $\mathrm{InO}_{x}$ samples. Gantmakher and collaborators [54] reported a similar behavior. The resistances of films, which have dropped significantly at high temperatures and appear to be superconducting, were found to saturate at nonzero values in the $T \rightarrow 0$ limit. This metallic regime, between the superconductor and insulator, exhibits a resistance much lower than that of the normal state. Chevernak and Valles in their magnetic field-tuned studies refer to this phase as a vortex liquid[86]. Tewari has suggested that this is a sharp crossover from a strong superconductor to an inhomogeneous state, which is a weak superconductor [88]. Kapitulnik et al. discussed the possibility of coupling to a dissipative heat bath characterized by a single parameter [89]. Dissipation plays a role in phase-only theories of the SIT of granular superconductors that build on the physics of resistively shunted Josephson junction arrays [90]. Dissipation can localize the phase of the system, leading to superconductivity [91] an effect reported for Josephson junction arrays [92]. Mason and Kapitulnik also reported that a ground plane in proximity with a
MoGe film seems to promote superconductivity, although it did not change the dynamical critical exponent as might be expected with enhanced screening [82]. Vishwanath et al. have presented a comprehensive theoretical discussion of screening and dissipation. In their work, a ground plane introduces screening of the long-range Coulomb interaction and provides a source of dissipation due to the gapless diffusive electrons in the plane [93].

Work on hot electron effects in the parallel field SIT of $a$ - $\mathrm{Bi}$, suggests that resistance saturation may in certain instances be the result of a failure of electrons to cool below a characteristic temperature, because ohmic dissipation heats the system of electrons relative to the lattice [94]. The analysis in this work also raises questions about the use of electric field scaling as a means of determining the exponent product $\nu(z+1)$ as demonstrated by Yazdani and Kapitulnik[53]. The idea is that the nonlinear regime subjected to electric field scaling competes with electron heating and for a certain range of parameters is the dominant effect. For other values, heating may be dominant. The essential feature of the nonlinear response is that the resistance depends upon current, which at constant current, corresponds to a value of the in-plane electric field. However if heating is the dominant effect and the electron lattice relaxation effects are weak, which may be the case at low temperatures, one can use $R(T)$ as a thermometer. Then each value of the measured resistance would correspond to an electron temperature. With the refrigerator temperature held constant, then only effect of increase current would be to heat the film's electrons. One can then map electric field scaling onto temperature scaling, obtaining the same parameters for the latter as when it is carried out directly. The success of this procedure suggests that electric field scaling works for $a$-Bi films because it is effectively temperature scaling. Thus it cannot be used to separately determine $v$ and $z$. The question is whether it is a general result.

As mentioned above, in the limit of zero temperature, nonlinear transport effects compete with Joule heating, with material specific properties determining the behavior. The electron temperature varies with power $P$ as $T_{\text {electron }} \sim P^{1 / \vartheta}$ with $\vartheta=p+2$ where $p$ is the power of temperature of the inelastic electron-phonon scattering rate, $\tau_{\text {in }}^{-1} \sim T^{p}$. Sondhi et al. [73] have presented a criterion for the dominance of Joule heating over nonlinear effects which is of the form of an inequality given by $2 / \vartheta<z /(z+1)$. This criterion arises from an argument based on dimensional analysis that may omit important multiplicative factors. Although we do not know $z$, we can assume it to be 1 or 2 . Then the value of $\vartheta \approx 5.56$ found for $a-\mathrm{Bi}$, together with the assumed values of $\mathrm{z}$ meets this criterion and heating would be the dominant effect [94].

Even in the earlier work of Yazdani and Kapitulnik on MoGe films, in which scaling analyses revealed apparent value of $\nu z \sim 1.35$ and $\nu(z+1) \sim 2.65$, which yielded $\nu \sim 1.3$ and $z \sim 1$, electron heating may be responsible. It is known that $p=2$ for MoGe so that $\vartheta=4$. This places 
MoGe in the "marginally dangerous category" in which both heating and nonlinear effects may be important. In fact, this value of $\vartheta$ implies that $V \sim T^{2}$ resulting in the direct mapping of electric field scaling onto temperature scaling as $V^{-1 / 2.65} \sim T_{\text {electron }}^{-2 / 2.65}$. This analysis assumes that electron heating may be responsible for the apparent electric field dependence of the resistance. As a consequence, the experimental support for the widely held belief that the dynamical critical exponent is unity is actually quite weak.

In studies of the thickness-dependent SIT of granular ultrathin films, a metallic regime is indeed found before the transition to zero resistance $[50,95,96]$. In contrast with the results mentioned above, this metallic regime is found at relatively high temperatures. Das and Doniach[97], Dalidovich and Phillips[98], and Ng and Lee [99], have presented theoretical approaches to intervening metallic phases. Phillips and Dalidovich have reviewed the subject in a comprehensive manner[100].

\section{Insulating Regimes of Disordered Superconduc- tors}

There has been considerable interest in the insulating regime attained upon the application of a perpendicular magnetic field. Palaanen, et al. [75], were the first to report a peak in $R(B)$ above the critical field of $\mathrm{InO}_{x}$ films. The Hall effect also became measurable at values of field near this field[101]. They suggested this was a signature of a transition or crossover between Bose and Fermi insulators and that the order parameter amplitude was non-vanishing in the insulating regime. More recently there have been a number of papers confirming and extending this work $[54,102,103,104,105,106]$. An alternative to the Bose-to-Fermi insulator crossover at this field is that the insulating regime consists of intermixed superconducting and normal phases. The understanding of this phenomenon has attracted considerable attention as Steiner, Boebinger and Kapitulnik have noted the similarity of data on some $\mathrm{InO}_{x}$ films to observations on cuprate superconductors, but with a much larger magnetic field scale for the latter[9]. They suggested that the insulating regime of cuprates attained in high fields is a Bose insulator. The conductivity can actually appear to fall to zero near criticality at sufficiently low temperatures, as reported by Sambandamurthy et al. [102] for $\mathrm{InO}_{x}$ films and for TiN films by Baturina et al. [103]. The explanation of this apparent "superinsulating" behavior in terms of a macroscopic Coulomb blockade in large Josephson junction arrays $[107,108,109]$ has generated significant controversy [110]. Recent work has shown that the switching between a highly insulating state and a conductive state may in part be a nonlinear thermal effect $[111,112]$. Other support for the existence of Cooper pairs in the insulating regime are the measurements of Markovic et al. [113], of the anisotropic magnetoresistance of films on the insulating side of the disorder-tuned SI transition, which support

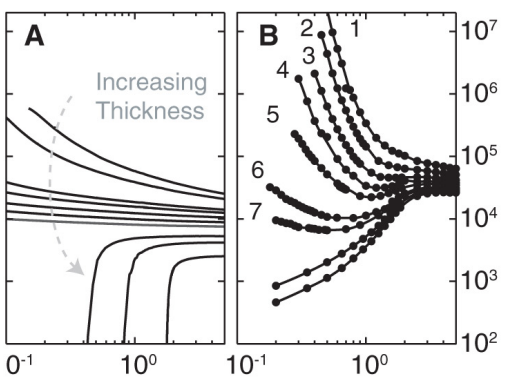

Temperature (K) Temperature $(\mathrm{K})$

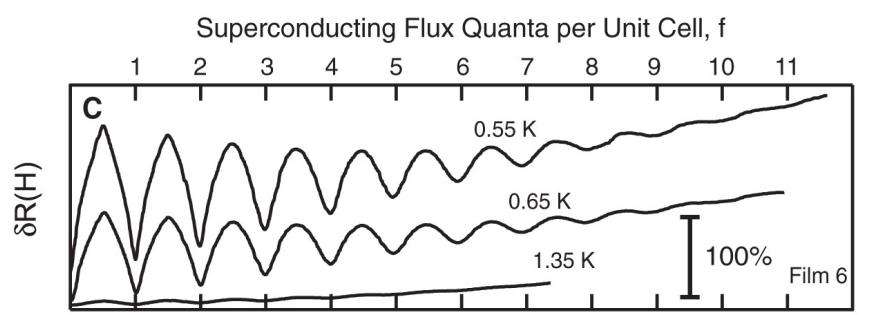

Figure 5: Electronic transport. (A) Transition from insulator to superconductor as a function of thickness of unpatterned $a-\mathrm{Bi} / \mathrm{Sb}$ films produced simultaneously with films, which are nano-honeycombed (NHC). From top to bottom, $d_{B i}=0.57,0.59,0.63,0.65,0.67,0.69$, $0.7,0.74,0.81$, and $0.95 \mathrm{~nm}$. There is no deviation in this data from that in the literature. (B) Similar transition of $a-\mathrm{Bi} / \mathrm{Sb}$ NHC films with $d_{B i}=1.09,1.13,1.16,1.17,1.20,1.24,1.27,1.3$, and $1.32 \mathrm{~nm}$ from top to bottom. Lines are guides to the eye. (C) Normalized magnetoresistance oscillations for one of the NHC films (no. 6) at three different temperatures. The frustration axis has been normalized by $h / 2 e S$, where $S$ is the area of a unit cell (see Fig. 5C). (From Ref. [13])

the idea that vortices are present. Gantmakher et al. concluded from an analysis of magneto-transport in $\mathrm{Cd}-\mathrm{Sb}$ alloys that there may be localized pairs in the insulating state of this system[114]. Crane et al. [14], reported studies of the complex AC conductivity of $\mathrm{InO}_{x}$ films through the magnetic field-tuned SIT. The data revealed a nonzero-frequency superfluid stiffness well into the insulating regime. Nernst effect measurements on the perpendicular field driven transition of $\mathrm{Nb}_{x} \mathrm{Si}_{1-x}$ films also suggest the presence of superconducting coherence in the insulating regime $[16,17,18]$.

One very serious issue has to do with the apparent absence of a giant magnetoresistance peak in most experiments involving quench-deposited films, whereas one is found almost always in studies of $\mathrm{TiN}$ and $\mathrm{InO}_{x}$. The first experiment on quench-evaporated films to observe a peak was that of Stewart et al., who presented results on quenchcondensed $a$-Bi films patterned with a nano-honeycomb array of holes on an alumina substrate [13]. Insulating films exhibited temperature-dependent resistances and magnetoresistance oscillations in the insulating regime that were dictated by the flux quantum $\mathrm{h} / 2 \mathrm{e}$, which were interpreted as of evidence of Cooper pairing in the insulating regime. This is shown in Fig. 5.

First we consider a few of the scenarios in which a mag- 
netoresistance peak is found. Shimshoni et al. [115], and Sheshadri et al. [116], suggested that the SIT is percolative. This phenomenology would become applicable if films were either physically inhomogeneous or, if actually homogenous, were rendered inhomogeneous by order parameter amplitude fluctuations [117]. This was found using a simple model of the disorder-tuned SIT involving a two-dimensional $s$-wave superconductor in the presence of a random potential with varying disorder strength. It was found that with increasing disorder, the pairing amplitude became spatially inhomogeneous. In the highdisorder regime the system would break up into superconducting islands with large pairing amplitude, separated by insulating regimes. The essential idea is that with increasing disorder on a microscopic scale the system selforganizes into a nanoscale granular structure in terms of a local-pairing amplitude. Related approaches consider the effect of the magnetic field on inducing islands that yield a magnetoresistance peak $[10,118]$. These scenarios blur the distinction between granular and homogenous systems, in that homogeneously disordered systems can self-organize into islands. In this picture, as a system is cooled, superconductivity is established in two steps. First the islands become superconducting, without phase coherence between them, and then as the temperature is lowered phase coherence and zero resistance sets in. In an early work of planar tunneling measurement, Barber et al. showed a non-zero pair amplitude in the insulating state of granular Pb films[119]. Generalizations of this picture[120] can in principle explain the observation of nonzero pairing amplitudes by both planar [70] and STM [71] tunneling experiments in the insulating regime of nominal homogeneous $\mathrm{InO}_{x}$ films. An example of this result for planar tunneling is shown in Fig. 6 in which an energy gap is observed in both superconducting and insulating $\mathrm{InO}_{x}$ films. Presumably experiments in the insulating regime of the field-tuned SIT would yield a similar result.

Recent experimental works suggest that variations of thickness on mesoscopic length scales are essential to the observation of the iconic magnetoresistance peak. The alumina nano-honeycomb substrates resulting in local phase coherence in the insulating regime shown in Fig. 5 are quit rough. In contrast, films grown on smooth Si substrates, which are patterned with a nano-honeycomb of holes do not exhibit oscillations in the insulating regime [121]. This result is different from previous observations of oscillations persisting in the insulating phase of films grown on nanohoneycomb arrays formed from alumina. The conclusion was that there are at least two distinct superconductorinsulator transitions, those in which Cooper pair phase coherence manifests itself in the insulating regime and those in which it does not.

The role of thickness fluctuations on local Cooper pair coherence and the giant MR peak is supported by the work of Lin and Goldman [122] and the work of Lin, Nelson and Goldman [123]. The focus of these works was on $a$-Bi films grown on $a$-Sb underlayers. When thick underlayers
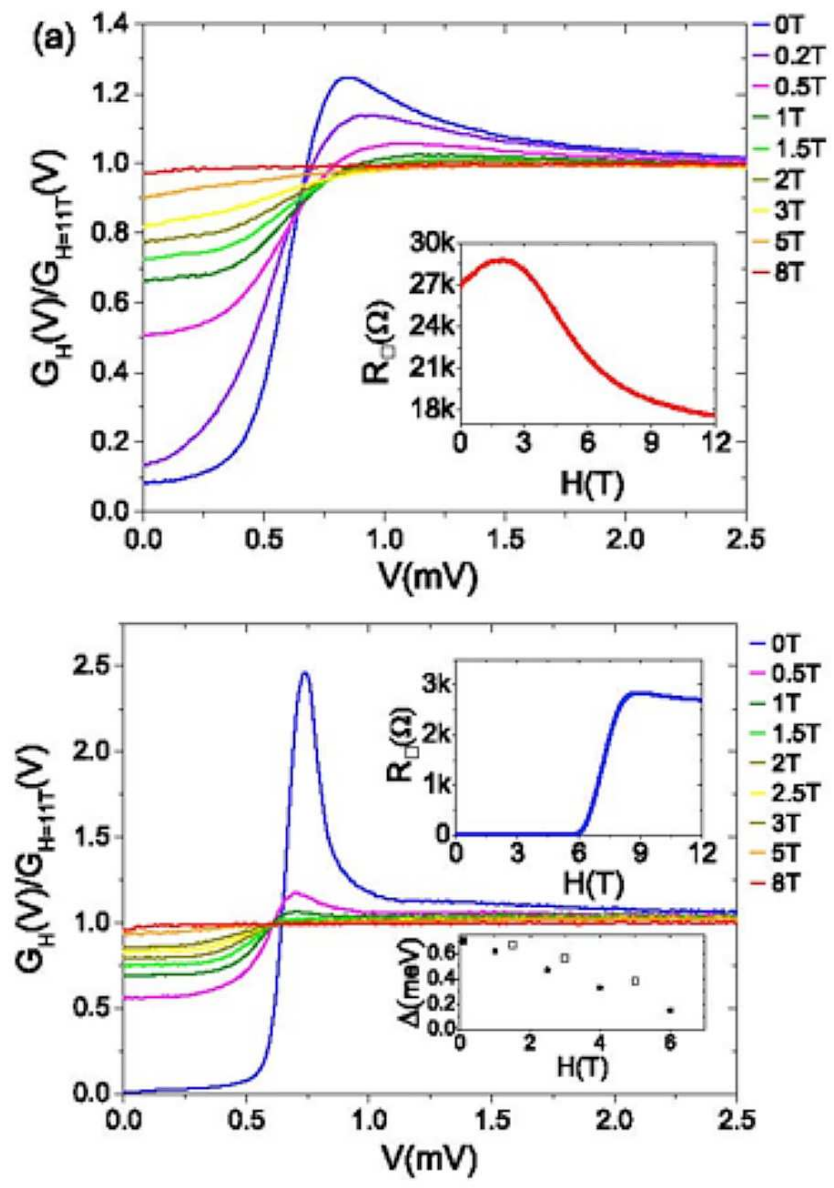

Figure 6: . (a) Normalized tunneling density of states obtained at $T=0.6 \mathrm{~K}$ and at different magnetic fields for an insulating $\mathrm{InO}_{x}$ sample, I. Inset: The corresponding magnetoresistance. (b) Normalized tunneling density of states obtained at $0.1 \mathrm{~K}$ and at different magnetic fields for a superconducting $\mathrm{InO}_{x}$ sample, S. Upper inset: The corresponding magnetoresistance for sample S. Lower inset: energy gap, $\Delta$ versus $H$ for sample I (empty black squares) and sample $\mathrm{S}$ (full black circles), obtained from a fit to the BCS expression for the density of states. (from Ref. [70])

were used, MR peaks were present. Post-growth atomic force microscope images indicated that films with thick underlayers were quite rough on nanometer scales, whereas films with underlayers with thicknesses less than $1 \mathrm{~nm}$ were smooth. The later did not exhibit MR peaks. This is detailed in the work of Lin, Nelson and Goldman.

Thickness fluctuations may be a type of disorder which is responsible for the properties of amorphous $\mathrm{InO}_{x}$ films as well. Atomic force microscope (AFM) images of films exhibiting magnetoresistance peaks indicate quite rough surfaces on mesoscopic length scales, films with out a peak are significantly smoother. Roughness could be interpreted as effective granularity. Other possible sources of disorder could be mesoscopic scale fluctuations in the In/O chemical ratio. Recent work by Givan and Ovadyahu [124] addresses just this issue. A microstructural study of amorphous $\mathrm{InO}_{x}$ films using high-resolution chemical analysis revealed spatial fluctuations on mesoscopic length scales of 
the $\mathrm{In} / \mathrm{O}$ ratio. Thus even films that are amorphous and exhibit well-behaved transport properties may be effectively disordered by compositional fluctuations on mesoscopic scales. These in turn can lead to mesoscopic scale carrier concentration fluctuations, and when superconductivity is present to strong pairing amplitude fluctuations. Whether the spatial variation of the $\mathrm{In} / \mathrm{O}$ ratio correlates with the spatial variation of thickness fluctuations is an open question.

The hypothesis that the behavior of $\mathrm{InO}_{x}$ is effectively inhomogeneous as borne out by several tunneling experiments. Also similar issues may arise in determining the properties of TiN films. The bottom line is that the giant MR peak and Arrhenius behavior of the resistance near it may be found in systems in which there are large fluctuations in the pair amplitude. In some theories they arise spontaneously, but experimentally they appear to be helped by thickness and/or compositional variations on a mesoscopic scale.

\section{SITs of Interfacial Superconductors}

In recent years the issue of SITs has played a role in the physics of the two-dimensional electron gases that form at the interfaces between insulating oxides. These systems have rich phase diagrams with a variety of ground states, some of which are superconducting. Furthermore their properties can be tuned by electrostatic charging [24, 25] permitting exploration of the quantum phase transitions between various ground states, such as the SIT.

Thus far there have been two prominent examples of SITs involving interfaces, between band insulators in the case of the $\mathrm{LaAlO}_{3} / \mathrm{SrTiO}_{3}$ system [24] and between a Mott insulator and a band insulator in the case of the $\mathrm{LaTiO}_{3} / \mathrm{SrTiO}_{3}$ system [25]. In both instances the interface was formed by vapor deposition of either $\mathrm{LaAlO}_{3}$ or $\mathrm{LaTiO}_{3}$ on a $\mathrm{TiO}_{2}$ terminated $\mathrm{SrTiO}_{3}$ substrate provided with a back gate. Thus the substrate serves as the gate dielectric for the field effect transistor structure. Charge trapping and hysteresis can occur so care must be taken in the charging process. Figure 7 shows the field-effect modulation of the transport properties of the interface.

Caviglia et al. [24] found that $R(T)$ in the superconducting regime was consistent with the Berezinskii-KosterlitzThouless (BKT) behavior expected for a two-dimensional system [35]. In the BKT picture the resistance above the transition temperature, $T_{B K T}$, is given by

$$
R \propto \exp \left[-\frac{b_{R}}{\left(T-T_{B K T}\right)^{1 / 2}}\right]
$$

where $b_{R}$ is a constant parameter related to properties of the vortices, which are material dependent.

They used this model to map out the phase diagram. From detailed data they showed that that near the range of gate voltages near the quantum critical point that the change in the induced carrier concentration, $\delta n_{2 D}$ was a linear function of the gate voltage, such that $\delta n_{2 D} \propto \delta V=$ $V-V_{C}$. Here $V_{C}$ is the gate voltage at criticality and $\delta n_{2 D}=n_{2 D}-n_{2 D_{C}}$ where $n_{2 D_{C}}$ is the areal carrier density at the critical point. Thus the gate voltage could be taken as the independent variable in the analysis of the quantum phase transition. The phase transition line in the superconducting regime from the scaling theory is then given by $T_{B K T} \propto\left(\delta n_{2 D}\right)^{\nu z} \propto(\delta V)^{\nu z}$. Here $z$ is the dynamical critical exponent and $\nu$ is the correlation length exponent. From a detailed analysis, of the dependence of $T_{B K T}$ on the gate voltage, they found the product $\nu z=2 / 3$, a value found for scaling analyses on several other systems, discussed earlier.

Similar studies were carried out by Biscaras et al. on $\mathrm{LaTiO}_{3} / \mathrm{SrTiO}_{3}$ interfaces [25]. This interface differs from the previous case in that $\mathrm{LaTiO}_{3}$ is an antiferromagnetic Mott insulator rather than a band insulator. The electron gas in this instance was shown to extend a few unit cells into the $\mathrm{SrTiO}_{3}$ layer. Concurrent measurements of the Hall effect reveal the presence of two types of carriers, which necessitated a complex analysis of the transport properties. Also the transport in the plane was found to be anisotropic. Subsequent work by this group involved a detailed scaling analysis, which yielded in an exponent product of $3 / 2$ for data in the low temperature limit.

In these works on interfacial superconductivity there is relatively little discussion or analysis of the insulating regime. The sheet resistances are significantly lower at low temperatures than those found for disordered thin films whose SITs are tuned any of the standard control parameters. If the curves of resistance vs. temperature are not activated, then the actual "insulating" regime may be that of a quantum-corrected metal. Such behavior is often found when less disordered amorphous films are driven out of the superconducting state by a magnetic field. Of course in the zero temperature limit a quantum corrected metal has zero conductance.

The interface between a superconductor and a band insulator can also show enhanced superconductivity. Although not directly related to the SIT, this phenomenon is an example of the great scientific opportunities that the study of interfaces can provide. A monolayer of FeSe grown on $\mathrm{SrTiO}_{3}$ exhibited a $T_{c}>100 \mathrm{~K}$ while bulk FeSe has $T_{c}=8 \mathrm{~K}[125]$. The critical temperature of the film is both larger than any other Fe based superconductor and is the only example of a maaterial that is not a cuprate with a critical temperature above the boiling point of liquid nitrogen. Measurements of the band structure show a single unit cell film to be heavily electron doped compared to the bulk[126]. Coupling to the $\mathrm{SrTiO}_{3}$ oxygen phonon modes was proposed as a possible mechanism for the enhanced $T_{c}[126]$. The bosonic modes driving up on temperature the superconductivityin FeSe would then be spatially separated from the FeSe layer. In this instance the underling mechanisms for superconductivity in the bulk and at the interface with $\mathrm{SrTiO}_{3}$ are certainly different. 

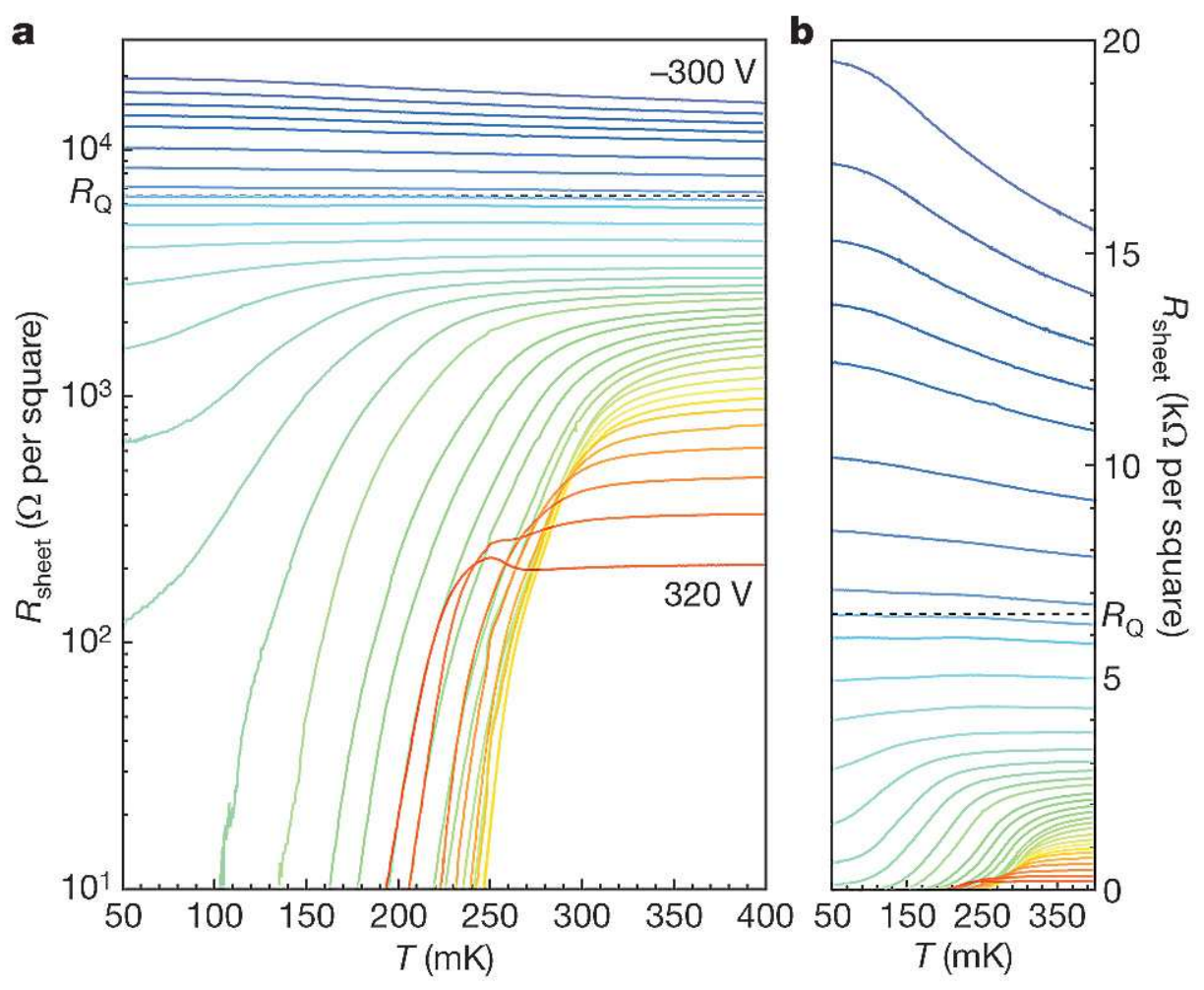

Figure 7: Field-effect modulation of the transport properties of a $\mathrm{LaAlO}_{3} / \mathrm{SrTiO}_{3}$ interface. (a) Measured sheet resistance as a function of temperature plotted on a semi-logarithmic scale, for gate voltages varying in $10-\mathrm{V}$ steps between $-300 \mathrm{~V}$ and $-260 \mathrm{~V}$, 20-V steps between -260 $\mathrm{V}$ and $320 \mathrm{~V}$, and for $-190 \mathrm{~V}$. The dashed line indicates the quantum of resistance $R_{Q}$. (b) The same data plotted on a linear resistance scale. (From Ref. [24])

\section{SITs of High Temperature Superconductors}

Electrostatic tuning is perhaps the simplest approach to studying the phase diagram of high-temperature superconductors and in particular traversing the SIT in such systems. Because the subject of electrostatic tuning is in its infancy, we will restrict our discussion to a brief account of recent results. The approach has the promise of simplifying the study of such systems and perhaps settling questions whose answers remain controversial. The key to success in this arena rests on two developments. The first is the ability to produce high quality ultrathin films of the cuprates by molecular beam epitaxy or high pressure oxygen sputtering. The second is the replacement of gating using a high dielectric constant crystal as both a substrate and a gate insulator with gating using ionic liquids. Using $\mathrm{SrTiO}_{3}$ substrates, charge transfers of order $10^{13}$ carriers $/ \mathrm{cm}^{2}$ are possible. With ionic liquids levels of charge transfer in excess of $10^{15}$ carriers $/ \mathrm{cm}^{2}$ have been realized. This level of charge transfer in principle would allow exploration of the full range of behaviors in the phase diagram.

Ionic liquids are liquid compounds at room temperature composed of ionic molecules, similar to molten salts. When incorporated in a field effect transistor configuration and gated, positive ions diffuse towards the negative elec- trode and negative ions to the positive electrode. Electric double layers form at the electrodes and behave as capacitors with nanometer separation between the ions and the induced charge. This nanometer spacing is what is responsible for the large areal charge transfer but this requires charging at a temperature where the ions are mobile. Typically a temperature somewhat below that at which solidification begins is chosen to suppress chemical reactions with and degradation of the electrode (cuprate). Because the induced charge at the electronic double layer occupies a thickness the order of the Fermi length in the material, it is important that the material to which charge is being transferred be a very thin. If it is not, then although one may be able to alter the charge near the surface, the deeper lying layers will be unmodified and the proximity effect will come into play greatly restricting the extent to which the properties of the film can be modified.

Exploiting such large charge transfers, this approach has been used to tune the SIT of $\mathrm{La}_{2-x} \mathrm{Sr}_{x} \mathrm{CuO}_{4}$ (LSCO) [21], of $\mathrm{YBa}_{2} \mathrm{Cu}_{3} \mathrm{O}_{7-x}$ (YBCO) [22] and more recently, that of oxygenated and superconducting $\mathrm{La}_{2} \mathrm{CuO}_{4+\delta}$ $(\delta-\mathrm{LCO})[23] . \quad$ In each of these studies the ionic liquid N, N-diethyl-N-(2-methoxyethyl)-N-methylammonium bis(trifluoromethyl sulphonyl)-imide (DEME-TFSI) was employed. Parenthetically, the use of electrochemical techniques to control the electronic properties of materials is 

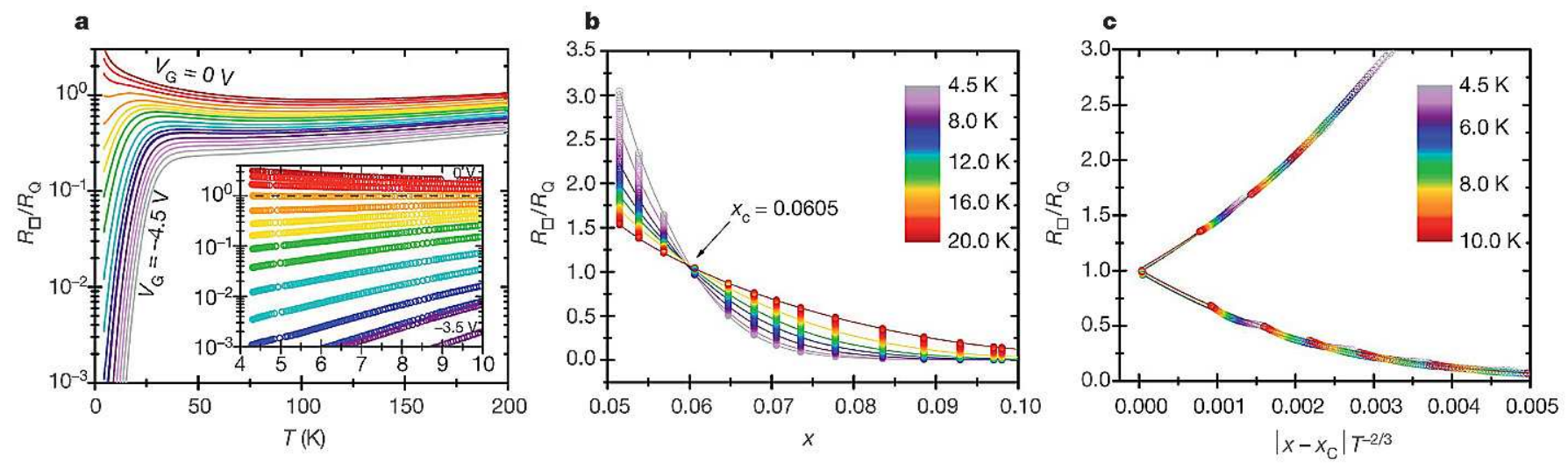

Figure 8: Superconductor-insulator transition driven by electric field. (a)Temperature dependence of normalized resistance $r=R_{\square}(x, T) / R_{Q}$ of an initially heavily underdoped and insulating film. Here $x$ is the number of carriers per $\mathrm{Cu}$. The device employs a coplanar Au gate and DEME-TFSI ionic liquid. The carrier density, fixed for each curve, is tuned by varying the gate voltage from $0 \mathrm{~V}$ to $24.5 \mathrm{~V}$ in $0.25 \mathrm{~V}$ steps; an insulating film becomes superconducting. The inset highlights a separatrix independent of temperature below $10 \mathrm{~K}$. The open circles are the actual raw data points; the black dashed line is $R_{\square}\left(x_{c}, T\right)=R_{Q}=6.45 k \Omega$. (b) The inverse representation of the same data, that is, the $r_{T}(x)$ dependence at fixed temperatures below $20 \mathrm{~K}$. Each vertical array of (about 100) data points corresponds to one fixed carrier density, that is, to one $r_{x}(T)$ curve. The colors refer to the temperature, and the continuous lines are interpolated for selected temperatures (4.5, $6.0,8.0,10.0,12.0,15.0$ and $20.0 \mathrm{~K})$. The crossing point defines the critical carrier concentration $x_{c}=0.06 \pm .01$, and the critical resistance $R_{c}=6.45 \pm 0.10 k \Omega$. (c) Scaling of the same data with respect to the variable $u=\left|x-x_{c}\right| T^{-1 / \nu z}$ with $\nu z=1.5$. For $4.3 K<T<10 K$, the discrete groups of points collapse accurately onto a two-valued function, with one branch corresponding to $x$ larger and the other to $x$ smaller than $\mathrm{s} x_{c}$. (Adapted from Ref. [21])

actually not particularly new, having been first done by Brattain and Garrett [127] in the 1950s. Its first use to control superconductivity was by McDevitt and collaborators [128] about 20 years ago. Here we will focus on the work of the Bozovic group on LSCO.

The work on LSCO involved the synthesis of epitaxial films that were one unit cell in thickness. This one unit cell thick film was grown on top of a buffer layer of insulating LCO. Many curves of $R(T)$ at different gate voltages were recorded and the SIT was traversed with detailed enough data to carry out an accurate scaling analysis. Figure 8 shows the result of the study of an underdoped and insulating film.

These remarkable experimental results are at first glance consistent with the predictions of the bosonic picture in a form in which the insulating regime is the electromagnetic dual of the superconducting regime. The fact that the critical resistance is $h / 4 e^{2}$ would seem to support this view. Also the exponent product $\nu z>1$. Together these would imply the presence of Cooper pairs on both sides of the quantum phase transition. The authors claim that this critical resistance is found only when the film thickness is precisely one unit cell.

There are several reasons to approach such a conclusion with caution. First the scaling analysis only involves data down to $T=4.3 \mathrm{~K}$, and there are indications of local minima in the resistance vs. temperature at the lowest temperatures. This implies that the scaling might break down at lower temperatures than those of these measurements. Second, it is hard to visualize a scenario in which the interaction energy between Cooper pairs is logarithmic in their separation, which would be the charge coun- terpart of the vortex interaction energy being logarithmic in the separation of vortices. Third, in studies of the SIT of four-unit cell thick $\delta$-LCO films there is a clear signature at high temperatures in the Hall effect that is found at charge transfers identical to those corresponding to the SIT in that system [23]. This implies that the transition is electronic in nature. It is of course possible that the ionic liquid charge tuned SITs in LSCO and $\delta-\mathrm{LCO}$ are different. Finally, in some systems, it has been reported that ionic liquids can favor the formation of oxygen vacancies, which can effectively act as chemical rather than electrostatics doping[129].

\section{Open Questions Relating to the SIT}

We have briefly reviewed the major results relating to the SIT in two dimensions, including interfacial systems as well as high temperature superconductors in addition to the consideration of nominally homogeneous disordered thin films. Despite a long history it is clear that there are many open questions relating to the SIT. These include, but are not limited to the following: What different physical models govern the various SITs of drastically different materials with different tuning parameters, which can have different exponent products and different critical resistances? Although there has been considerable progress there is still a question as to why the insulating states of various transitions are so very different. What is the correct theory of the ubiquitous large magnetoresistance peak? Is this phenomenon one in which there are localized Cooper pairs in an essentially homogenous system, or in particular is disorder on a length scale greater 
than atomic scales necessary for such a peak? Under what conditions is an intermediate metallic regime, or a twophase regime a feature of a particular SIT? Finally, how does electrostatic tuning of the carrier concentration differ from chemical tuning? A comprehensive review of the SIT problem with a more detailed discussion of the theories associated with the behavior of disordered superconductors is that of Gantmakher and Dogolpolov [130].

Finally, although the work described in detail here has no direct bearing on the issue of raising the superconducting critical temperature, some of the procedures employed may have relevance. Certainly ionic liquid gating may facilitate the search for new superconductors through bypassing the complexities of chemical doping for which a separate sample is required for each doping level. Also interfacial stuudies may be an important route to high critical temperatures with the work on FeSe serving as an existence proof for the concept [125]. In some manner this could lead to a realization of the ideas of Ginzburg and Little in which a conductive layer derives its superconducting nature from a separate layer or layers in intimate contact with it [131].

The authors would like to thank Nandini Trivedi, Aviad Frydman and Zvi Ovadyahu for helpful discussions. This work was supported by the National Science Foundation under Grant No. NSF/DMR-0854752 and NSF/DMR1263316. Part of this work was carried out at the University of Minnesota Characterization Facility, a member of the NSF-funded Materials Research Facilities Network via the MRSEC program, and the Nanofabrication Center which receives partial support from the NSF through the NNIN program.

\section{References}

[1] Subir Sachdev. Quantum Phase Transitions, 2nd ed., Cambridge University Press, Cambridge, 2011.

[2] A. Gerber, A. Milner, G. Deutscher, M. Karpovsky, A. Gladkikh, Insulator-superconductor transition in 3D granular films, Phys, Rev. Lett. 78 (1997) 4277.

[3] H. S. J. van der Zant, W. J. Elion, L. J. Geerligs, J. E. Mooij, Quantum phase transitions in two dimensions: experiments in Josephson junction arrays, Phys. Rev. B 54 (1996) 10081.

[4] Christof Bruder, Rosario Fazio, Gerd Schon, The BoseHubbard model from Josephson junction arrays to optical lattices. Ann. Phys. (Leipzig), 9-10 (2005) 566.

[5] G. Sambandamurthy, L. W. Engel, A. Johansson, D. Shahar, Experimental Evidence for a Collective Insulating State in Two-dimensional Superconductors, Phys. Rev. Lett. 94 (2005) 017003.

[6] T. I. Baturina, A. Yu. Mironov, V. M. Vinokur, M. R. Baklanov, C. Strunk, Localized superconductivity in the quantum-critical region of the disorder-driven superconductor-insulator-transition in TiN thin films. Phys. Rev. Lett. 99 (2007) 257003.

[7] V. Yu. Butko, P. W. Adams, Quantum metallicity in a two-dimensional insulator, Nature 409 (2001) 161

[8] T. I. Baturina, C. Strunk, M. R. Baklanov, A. Satta, Quantum metallicity on the high-field side of the superconductor-insulator transition, Phys. Rev. Lett. 98 (2007) 127003.
[9] M. Steiner, G. Boebinger, A. Kapitulnik, Possible fieldtuned superconductor-insulator transition in high-Tc ssuperconductors: implications for pairing at high magnetic fields, Phys. Rev. Lett. 94 (2005) 107008.

[10] Yonatan Dubi, Yigal Meir, Yishai Avishai, Nature of the superconductor-insulator transition in disordered superconductors. Nature 449 (2007) 876.

[11] Y. Seo, Y. Qin, C. L. Vicente, K. S. Choi, Jongsoo Yoon, Origin of nonlinear transport across the magnetically induced superconductor-metal-insulator transition in two dimensions. Phys. Rev. Lett. 97 (2006) 057005 .

[12] Yongguang Qin, C. L. Vicente, Jongsoo Yoon, Magnetically induced metallic phase in superconducting tantalum films. Phys. Rev. B 73 (2006) 100505. [13]M. D. Stewart, Jr., Aijun Yin, J. M. Xu, James M. Valles Jr., Superconducting Pair Correlations in an Amorphous Insulating Nanohoneycomb Film, Science 318 (2007) 1273.

[13] M. D. Stewart, Jr., Aijun Yin, J. M. Xu, James M. Valles Jr., Superconducting Pair Correlations in an Amorphous Insulating Nanohoneycomb Film, Science 318 (2007) 1273.

[14] R. Crane, N. P. Armitage, A. Johansson, G. Sambandamurthy, D. Shahar, G. Gruner, Survival of superconducting correlations across the two-dimensional superconductor-insulator transition: a finite-frequency study, Phys. Rev. B 75 (2007) 184530.

[15] K. H. Sarwa B. Tan, Kevin A. Parendo, A. M. Goldman, Evidence of spatially inhomogeneous pairing on the insulating side of a disorder-tuned superconductorinsulator transition. Phys. Rev. B 78 (2008) 014506.

[16] A. Pourret, H. Aubin, J. Lesueur, C. A. MarracheKikuchi, L. Berge, L. Dumoulin, K. Behnia, Observation of the Nernst signal generated by fluctuating Cooper pairs, Nature Physics 2 (2008) 683.

[17] A. Pourret, H. Aubin, J. Lesueur, C. A. MarracheKikuchi, L. Berge, L. Dumoulin, K. Behnia, Length scale for the superconducting Nernst signal above Tc in Nb0.15Si0.85, Phys. Rev. B 76 (2007) 214504.

[18] P. H. Spathis, H. Aubin, A. Pourret, K. Behnia, Nernst effect in the phase-fluctuating superconductor $\mathrm{InOx}$, Europhysics Lett. 83 (2008) 57005.

[19] Kevin A. Parendo, K. H. Sarwa B. Tan, A. Bhattacharya, M. Eblen-Zayas, N. Staley, A. M. Goldman, Electrostatic tuning of the superconductor-insulator transition in two dimensions, Phys. Rev. Lett. 94 (2005) 197004.

[20] Kevin A. Parendo, K. H. Sarwa B. Tan, K. H. A. M. Goldman, Electrostatic and parallel-magnetic-field tuned two-dimensional superconductor-insulator transitions. Phys. Rev. B 73 (2006) 174527.

[21] A. T. Bollinger, G. Dubuis, J. Yoon, D. Pavuna, J. Misewich, I. Bozovic, Superconductor-insulator transition in $\mathrm{La}_{2-x} \mathrm{Sr}_{x} \mathrm{CuO}_{4}$ at the pair quantum resistance, Nature 472 (2011) 458.

[22] Xiang Leng, Javier Garcia-Barriocanal, Shameek Bose, Yeonbae Lee, A. M. Goldman, Electrostatic Control of the Evolution from a Superconducting Phase to an Insulating Phase in Ultrathin $\mathrm{YBa}_{2} \mathrm{Cu}_{3} \mathrm{O}_{7-x}$ Films, Phys Rev. Lett. 107 (2011) 027001.

[23] J. Garcia-Barriocanal, A. Kobrinskii, X. Leng, J. Kinney, B. Yang, S. Snyder, A. M. Goldman, Electronically driven superconductor-insulator transition in electrostatically doped $\mathrm{La}_{2} \mathrm{CuO}_{4+\delta}$ thin films, Phys. Rev. B 87 (2013) 024509

[24] A. D. Caviglia, S. Gariglio, N. Reyren, D. Jaccard. T. Schneider, M. Gabay, S. Thiel, G. Hammer, J. Mannhart, J.-M. Triscone, Electric field control of the $\mathrm{LaAlO}_{3} / \mathrm{SrTiO}_{3}$ interface ground state, Nature 456 (2008) 624. 
[25] J. Biscaras, N. Bergeal, S. Hurand, C. Grossetete, A. Rastogi, R. C. Budhani, D. LeBoeuf, C. Proust, J. Lesueur, Two-Dimensional Superconducting Phase in $\mathrm{LaTiO}_{3} / \mathrm{SrTiO}_{3}$ Heterostructures Induced by HighMobility Carrier Doping, Phys. Rev. Lett. 108 (2012) 247004.

[26] P. W. Anderson, Theory of Dirty Superconductors, J. Phys. Chem. Solids 11 (1959) 26.

[27] A. A. Abrikosov, L. P. Gor'kov, Superconducting alloys at non-zero temperatures, Zh. Eksp. Teor. Fiz.(USSR) 36 (1959) 319.

[28] P. A. Lee, T. V. Ramakrishnan, Disordered electronic systems. Rev. Mod Phys. 57 (1985) 287.

[29] A. I. Shal'nikov, Superconducting properties of thin Metallic Layers, Zh. Eksp. Teor. Fiz. 10, 630 (1940)

[30] W. Buckel, R. Hilsch, Influence of condensation at low temperatures on the electrical resistance and superconduction of various metals, Z. Phys. 138 (1954) 109.

[31] R. E. Glover, Ideal resistive transition of a Superconductor, Phys. Lett. 25A, 542 (1967)

[32] Myron Strongin, R. S. Thompson, O. F. Kammerer, and J. E. Crow, Destruction of Superconductivity in Disordered Near-Monolayer Films, Phys. Rev. B 1, 1078 (1970)

[33] R. C. Dynes, J. P. Garno, and J. M. Rowell, Two-Dimensional Electrical Conductivity in QuenchCondensed Metal Films, Phys. Rev. Lett. 40, 479 (1978)

[34] M. Ma, P. A. Lee, Localized superconductors, Phys. Rev. B 32 (1985) 5658.

[35] J. E. Mooij, in Percolation, Localization and Superconductivity, eds. Allen M. Goldman and Stuart A. Wolf, (Plenum Press, New York and London, 1983), p. 325.

[36] Elihu Abrahams, Sergey V. Kravchenko, Myriam P. Sarachik, Metallic behavior and related phenomena in two dimensions, Rev. Mod. Phys. 73 (2001) 251.

[37] S. Maekawa, H. Fukuyama, Localization effects in twodimensional superconductors, J. Phys. Soc. Japan 51 (1982) 1380.

[38] H. Ebisawa, H. Fukuyama, S. Maekawa, Superconducting transition temperature of dirty thin films in weakly localized regime, J. Phys. Soc. Japan 54 (1985) 2257.

[39] A. M. Finkel'stein, Suppression of superconductivity in homogeneously disordered systems, Zh. Eksp. Teor. Fiz. 45 (1987) 37 [Sov. Phys. JETP Lett. 45 (1987) 46]; Physica B 197 (1994) 636.

[40] Matthew P. A. Fisher, Quantum phase transitions in disordered two-dimensional superconductors, Phys. Rev. Lett. 65 (1990) 923.

[41] D. B. Haviland, Y. Liu, A. M. Goldman, Onset of superconductivity in the two-dimensional limit, Phys. Rev. Lett. 621(989) 2180.

[42] A. F. Hebard, M. A. Paalanen, Magnetic-field-tuned superconductor-insulator transition in two-dimensional films, Phys. Rev. Lett. 65 (1990) 927.

[43] A. Larkin, Superconductor-insulator transitions in films and bulk materials, Ann. Phys. (Leipzig) 8 (1999) 785.

[44] Y. M. Strelniker, A. Frydman, and S. Havlin, Percolation model for the superconductor-insulator transition in granular films, Phys. Rev. B 76 (2007) 224528.

[45] J. M. Graybeal, M. R. Beasley, Localization and interaction effects in ultrathin amorphous superconducting films, Phys. Rev. B 29 (1984) 4167.

[46] [Matthew P. A. Fisher, G. Grinstein, S. M. Girvin, Presence of quantum diffusion in two dimensions: Universal resistance at the superconductor-insulator transition, Phys. Rev. Lett. 64 (1990) 587.

[47] Min-Chul Cha, Matthew P. A.Fisher, S. M. Girvin, Mats Wallin, Peter Young, Universal conductivity of two dimensional films at the superconductor-insulator transition, Phys. Rev. B 44 (1991) 6883.

[48] S. Doniach, Quantum fluctuations in two-dimensional superconductors, Phys. Rev. B 24 (1981) 5063.

[49] X. G. Wen, A. Zee, Universal conductance at the superconductor-insulator transition, Int. J. of Mod. Phys. B 4 (1990) 437.

[50] H. M. Jaeger, D. B. Haviland, B. G. Orr, A. M. Goldman, Onset of superconductivity in ultrathin granular metal films, Phys. Rev. B 40 (1989) 182.

[51] Orr, B. G., Jaeger, H. M. and Goldman, A. M., Transition-Temperature Oscillations in Thin Superconducting Films, Phys. Rev. Lett. 53 (1984) 2046.

[52] Gou et al., Superconductivity Modulated by Quantum Size Effects, Science 306, (2004) 1915-1917.

[53] Ali Yazdani, Aharon Kapitulnik, Superconductinginsulating transition in two-dimensional a-MoGe thin films, Phys. Rev. Lett. 74 (1995) 3037.

[54] V. F. Gantmakher, M. V. Golubkov, V. T. Dolgopolov, G. E. Tsydynzhapov, A. A. Shashkin, sSuperconductorinsulator transition in amorphous In-O films, Physica B 284-288 (2000) 649 .

[55] N. Markovic, C. Christiansen, A. M. Goldman, Thickness-magnetic field phase diagram at the superconductor-insulator transition in 2D, Phys. Rev. Lett. 81 (1998) 5217.

[56] N. Markovic, C. Christiansen, A. M. Mack, W. H. Huber, A. M. Goldman, Superconductor-insulator transition in two dimensions, Phys. Rev. B 60 (1999) 4320.

[57] N. Markovic, A.. M. Mack, G. Martinez-Arizala, C. Christiansen, A. M. Goldman, Evidence of vortices on the insulating side of the superconductor-insulator transition, Phys. Rev. Lett. 81 (1998) 701.

[58] Myles Steiner, Nicholas P. Breznay, Aharon Kapitulnik, Approach to a superconductor-to-Bose-insulator transition in disordered films, Phys. Rev. B 77 (2008) 212501.

[59] Wenhao $\mathrm{Wu}$, private communication.

[60] A. Gold, Dynamical conductivity of the twodimensional Bose condensate: superfluid-insulator transition for a long-range random potential, Z. Phys B 87 (1992) 169.

[61] W. Krauth, N. Trivedi, D. Ceperley, Superfluidinsulator transition in disordered boson systems, Phys. Rev. Lett. 67 (1991) 2307.

[62] M. Makivic, N. Trivedi, S. Ullah, Disordered bosons: critical phenomena and evidence for new lo energy excitations, Phys. Rev. Lett. 71 (1993) 2307.

[63] Nandini Trivedi, Richard T. Scalettar, Mohit Randeria, Superconductor-insulator transition in a disordered electronic system, Phys. Rev. B 54 (1996) 3756(R).

[64] Karl-Heinz Wagenblast, Anne van Otterlo, Gerd Schon, Gergely T. Zimanyi, Superconductor-insulator transition in a tunable dissipative environment, Phys. Rev. Lett. 79 (1997) 2730.

[65] Philip Phillips, Denis Dalidovich, Electron-like quasiparticles drive the superconductor-insulator transition in homogeneously disordered thin films, Phil Mag. B 81 (2001) 847.

[66] J. M. Valles, Jr., R. C. Dynes, J. P. Garno, Superconductivity and the electronic density of states in disordered two-dimensional metals, Phys. Rev. B 40 (1989) 6680 .

[67] R. A. Smith, Michael Yu. Reizer, John W. Wilkins, Suppression of the order parameter in homogeneous disordered superconductors, Phys Rev.B 51 (1995) 6470.

[68] J. M. Valles, Jr., R. C Dynes, J. P. Garno, Electron tunneling determination of the order-parameter amplitude at the superconductor-insulator transition in $2 \mathrm{D}$, Phys. Rev. Lett. 69 (1995) 3567.

[69] Shih-Ying Hsu, J. A. Chervenak, J. M. Valles, Jr. , Magnetic field enhanced order parameter amplitude fluctuations in ultrathin films near the superconductorinsulator transition, Phys. Rev. Lett. 75 (1995) 132.

[70] D. Sherman, G. Kopnov, D. Shahar, A. Frydman, Mea- 
surement of a Superconducting Energy Gap in a Homogeneously Amorphous Insulator, Phys. Rev. Lett. 108 (2012) 177006.

[71] Benjamin Sacepe, Thomas Dubouchet, Claude Chapelier, Marc Sanquer, Maoz Ovadia, Dan Shahar, Mikhail Feigel'man, Lev Ioffe, Localization of preformed Cooper pairs in disordered superconductors, Nature Physics 7 (2011) 239 .

[72] J. S. Meyer, B. D. Simons, Gap fluctuations in inhomogeneous superconductors, Phys. Rev. B 64 (2001) 134516.

[73] S. L. Sondhi S. M. Girvin, J. P. Carini, D. Shahar, Continuous quantum phase transitions, Rev. Mod. Phys. 69 (1997) 315.

[74] Igor F. Herbut, Quantum critical points with the Coulomb interaction and the dynamical exponent: when and why $z=1$. Phys. Rev. Lett. 87 (2001) 137004.

[75] M. A. Paalanen, A. F. Hebard R. R. Ruel, Lowtemperature insulating phases of uniformly disordered two-dimensional superconductors, Phys. Rev. Lett. 69 (1992) 1604.

[76] S. Okuma N. Kokubo, Unusual insulating phase at low temperature in thin indium films, Phys. Rev. B 51 (1995) 15415.

[77] M. Inoue, H. Matsushita, H. Hayakawa, K. Ohbayashi, Critical sheet resistance and two-dimensional properties of $\mathrm{Bi}_{2} \mathrm{Sr}_{2} \mathrm{CuO}_{x}$ thin films, Phys. Rev. B 51 (1995) 15448.

[78] G. T. Seidler, T. F. Rosenbaum, B. W. Veal, Two-dimensional superconductor-insulator transition in bulk single-crystal $\mathrm{YBa}_{2} \mathrm{Cu}_{3} \mathrm{O}_{6.38}$, Phys. Rev. B 45 (1992) 10162.

[79] T. Wang, K. M. Beauchamp, D. D. Berkley, B. R. Johnson, J.-X. Liu, J. Zhang, A. M. Goldman, Onset of high temperature superconductivity in the two-dimensional limit, Phys. Rev. B 43 (1991) 8823.

[80] S. Okuna, T. Terashima, N. Kokubo, Anomalous magnetoresistance near the superconductor-insulator transition in ultrathin films of $a-\mathrm{Mo}_{x} \mathrm{Si}_{1-x}$, Phys. Rev. B 58 (1998) 2816.

[81] J. T. Chayes, L. Chayes, Daniel S. Fisher, T. Spencer, Finite-Size Scaling and Correlation Lengths for Disordered Systems. Phys. Rev. Lett. 57 (1986) 2999.

[82] Nadya Mason, Aharon Kapitulnik, Superconductorinsulator transition in a capacitively coupled dissipative environment. Phys. Rev. B 65 (2002) 220505(R).

[83] J. S. Parker, D. E. Read, A. Kumar, P. Xiong, Superconducting quantum phase transitions tuned by magnetic impurity and magnetic field in ultrathin $\mathrm{a}-\mathrm{Pb}$ films, Europhys. Lett. 75 (2006) 950.

[84] [Kevin A. Parendo, K. H. Sarwa B. Tan, A. Bhattacharya, M. Eblen-Zayas, N. Staley, A. M. Goldman, Tuning the 2D superconductor insulator transition by use of the electric field effect, AIP Conference Proceedings 850 (2006) 949s.

[85] Nadya Mason, Aharon Kapitulnik, Dissipation Effects on the Superconductor-Insulator Transition in 2D Superconductors. Phys. Rev. Lett. 82 (1999) 5341.

[86] J. A. Chervenak, J. M. Valles, Jr., Absence of a zerotemperature vortex solid phase in strongly disordered superconducting Bi films, Phys. Rev. B 61 (2000) 9245(R).

[87] Yongguang Qin, C. L. Vicente, Yongsoo Yoon, Magnetically induced metallic phase in superconducting tantalum films, Phys. Rev. B 73 (2006) 100505.

[88] Sumanta Tewari, Crossover and scaling in a twodimensional field-tuned superconductor. Phys. Rev. B 69 (2004) 014512

[89] Aharon Kapitulnik, Nadya Mason, Steven A. Kivelson Kivelson, Sudip Charkravarty, Effects of dissipation on quantum phase transitions. Phys. Rev. B 63 (2001)
125322 .

[90] I. V. Yurkevich, Igor V. Lerner, Granular superconductors: From the nonlinear $\sigma$ model to the Bose-Hubbard description. Phys. Rev. B 64 (2001) 054515.

[91] Albert Schmid, Diffusion and Localization in a Dissipative Quantum System, Phys. Rev. Lett. 51 (1983) 1506.

[92] A. J. Rimberg, T. R. Ho, C. Kurdak, John Clarke, K. L. Campman, A. C. Gossard, DissipationDriven Superconductor-Insulator Transition in a TwoDimensional Josephson-Junction Array. Phys. Rev. Lett. 78 (1997) 2632.

[93] Ashvin Vishwanath, Joel E. Moore, T. Senthil, Screening and dissipation at the superconductor-insulator transition induced by a metallic ground plane, Phys. Rev. B 69 (2004) 054507.

[94] Kevin A. Parendo, K. H. Sarwa Tan, A. M. Goldman, Hot-electron effects in the two-dimensional superconductor-insulator transition, Phys. Rev. B 74 (2006) 134517.

[95] C. Christiansen, L. M. Hernandez, A. M. Goldman, Evidence of Collective Charge Behavior in the Insulating State of Ultrathin Films of Superconducting Metals, Phys. Rev. Lett.. 88 (2002) 037004.

[96] [87]Kevin A. Parendo, K. H. Sarwa Tan, A. M. Goldman, Evolution towards superconductivity in granular films of bismuth, Phys. Rev. B 76 (2007) 100508.

[97] D. Das, S. Doniach, Existence of a Bose metal at $\mathrm{T}=$ 0, Phys. Rev. B 60 (1999) 1261.

[98] Denis Dalidovich, Philip Phillips, Phase Glass is a Bose Metal: A New Conducting State in Two Dimensions, Phys. Rev. Lett. 89 (2002) 027001.

[99] Ta Kai Ng, Derek K. K. Lee, Possibility of a metallic phase in granular superconducting films, Phys. Rev. B 63 (2001) 144509.

[100] Philip Phillips, Denis Dalidovich, The Elusive Bose Metal Science 302 (2003) 243.

[101] Matthew P. A. Fisher, Hall effect at the magneticfield-tuned superconductor-insulator transition Physica A 177 (1991) 553

[102] G. Sambandamurthy, L. W. Engel, A. Johansson, D. Shahar, Superconductivity-Related Insulating Behavior, Phys. Rev. Lett. 92 (2004) 107005.

[103] T. I. Baturina, A.Yu. Mironov, V. M. Vinokur, M. R. Baklanov, C. Strunk, Localized Superconductivity in the Quantum-Critical Region of the DisorderDriven Superconductor-Insulator Transition in TiN Thin Films, Phys. Rev. Lett. 99 (2007) 257003.

[104] Myles Steiner, Aharon Kapitulnik, Superconductivity in the insulating phase above the field-tuned superconductor-insulator transition in disordered indium oxide films, Physica C 422 (2005) 16.

[105] Y. J. Lee, Y. S. Kim, E. N. Bang, H. Lim, H. K.. Shin, Magnetoresistance of amorphous indium oxide films on the insulating side near the superconductor-insulator transition, J. Phys.: Condens. Mater. 13 (2001) 8135.

[106] Tatyana I. Baturina, D. R . Islamov, Z. D. Kvon, M. R. Baklanov, A. Satta, High-field side of SuperconductorInsulator Transition., arXivcond-mat/0210250.

[107] Valerii M. Vinokur, Tatyana I. Baturina, Mikhail V. Fistul, Aleksey Yu. Mironov, Mikhail R. Baklanov, Superinsulator and quantum synchronization. Nature 452 (2008) 613.

[108] M. V. Fistul, V. M. Vinokur, T. I. Baturina, Collective Cooper-Pair Transport in the Insulating State of Josephson-Junction Arrays, Phys. Rev. Lett. 100 (2008) 086805.

[109] M. V. Fistul, V. M Vinokur, T. I. Baturina, Macroscopic Coulomb blockade in large Josephson junction arrays, arXiv:0806.4311.

[110] K. B. Efetov, M. V. Feigel'man, P. B. Wiegmann, Comment on Superinsulator and Quantum Synchronization, 
arXiv:0804.3775v3.

[111] M. Ovadia, B. Sacepe, D. Shahar, Electron-Phonon Decoupling in Disordered Insulators, Phys. Rev. Lett. 102 (2009) 176802.

[112] B. Altschuler, V. E. Kravtsov, I. V. Lerner, I. L. Aleiner, Jumps in Current-Voltage Characteristics in Disordered Films, Phys. Rev. Lett. 102 (2009) 176803.

[113] N. Markovic, A. M. Mack, G. Martinez-Arizala, C. Christiansen, A. M. Goldman, Evidence of Vortices on the Insulating Side of the Superconductor-Insulator Transition, Phys. Rev. Lett. 81 (1998) 701.

[114] V. F. Gantmakher, Superconductor insulator transitions and insulators with localized pairs, PhysicsUspekhi 41 (1998) 214.

[115] Efrat Shimshoni, Assa Auerbach, Aharon Kapitulnik, Transport through Quantum Melts, Phys. Rev. Lett. 80 (1998) 3352.

[116] K. Sheshadri, H. R. Krishnamurthy, Rahul Pandit, T. V. Ramakrishnan, Percolation-Enhanced Localization in the Disordered Bosonic Hubbard Model, Phys. Rev. Lett. 75 (1995) 4075.

[117] Amit Ghosal, Mohit Randeria, Nandini Trivedi, Inhomogeneous pairing in highly disordered s-wave superconductors, Phys. Rev. B 65 (2001) 014501.

[118] Yonatan Dubi, Yigal Meir, Yshai Avishai, Unifying Model for Several Classes of Two-Dimensional Phase Transition, Phys. Rev. Lett. 94 (2005) 156406.

[119] R. P. Barber, Jr., L. M. Merchant, A. La Porta, and R. C. Dynes, Tunneling into granular $\mathrm{Pb}$ films in the superconducting and insulating regimes, Phys. Rev. B 49 (1994) 3409.

[120] Ksarim Bouadim, Yen Lee Loh, Mohit Randeria, Nandini Trivedi, Single- and two-particle energy gaps across the disorder-driven superconductor- insulator transition, Nature Physics 7 (2011) 884.

[121] S. M. Hollen, G. E. Fernandes, J. M. Xu, J. M. Valles Jr., Collapse of the Cooper pair phase coherence length at a superconductor-to-insulator transition, Phys. Rev. B 87 (2013) 054512.

[122] Yen-Hsiang Lin, A. M. Goldman, Magnetic-FieldTuned Quantum Phase Transition in the Insulating Regime of Ultrathin Amorphous Bi Films, Phys. Rev. Lett. 1106 (2011) 127003.

[123] Yen-Hsiang Lin, J. Nelson, A. M. Goldman, The role of mesoscopic disorder in determining the character of the field-induced insulating regime of amorphous ultrathin films, Physica C 497 (2014) 102.

[124] U. Givan, Z. Ovadyahu, Compositional disorder and transport peculiarities in the amorphous indium oxides. Phys. Rev. B 86 (2012) 86165101.

[125] Ge et al., Superconductivity above $100 \mathrm{~K}$ in single-layer FeSe films on doped $\mathrm{SrTiO}_{3}$, Nat. Mater. advanced online publication (2014).

[126] Lee et al., Interfacial mode coupling as the origin of the enhancement of $T_{c}$ in FeSe films on $\mathrm{SrTiO}_{3}$, Nature 515 (7526) (2014) 245-248.

[127] W. H. Brattain, C. G. B. Garrett, Experiments on the Interface between Germanium and an Electrolyte, Bell System Technical Journal 34 (1955) 129.

[128] Steven G. Haupt, David R Riley, Christopher T. Jones, Jinai Zhao, John T. McDevitt, Reversible modulation of Tc in conductive polymer/high temperature superconductor assemblies, J. Am. Chem. Soc. 115 (1993) 1196.

[129] Jaewoo Jeong, Nagaphani Aetukuri, Tanja Graf, Thomas D. Schladt, Mahesh G. Samant, Stuart S. P. Parkin, Suppression of Metal-Insulator Transition in VO2 by Electric Field-Induced Oxygen Vacancy Formation, Science 339 (2013) 1402.

[130] V. F. Gantmakher, V. T. Dolgopolov, Superconductorinsulator quantum phase transition, Physics- Uspekhi
$53(2010) 1$.

[131] W. A. Little, Vitaly Ginzburg and High Temperature Superconductivity, Journal of Superconductivity and Novel Materials, 19 (2006) 443. 